\title{
KAJIAN MATRIKS INTERVAL DAN SIFAT-SIFATNYA
}

\author{
Epifania Kurva, Evi Noviani, Nilamsari Kusumastuti
}

\begin{abstract}
INTISARI
Misalkan IR menyatakan himpunan semua interval tertutup atas $\boldsymbol{R}$. Matriks interval merupakan perluasan dari matriks real dengan elemen - elemen pada matriks interval berupa interval di IR. Aritmetika matriks interval merupakan generalisasi dari aritmetika matriks real, dimana operasinya menggunakan aturan-aturan yang berlaku dalam aritmetika interva. Oleh karena itu,terdapat sifat-sifat yang berlaku dalam aritmetika matriks real tapi tidak berlaku dalam aritmetika matriks interval. Sifatsifat tersebut terkait sifat distributif yang tidak selalu berlaku pada elemen di IR.
\end{abstract}

Kata kunci : matriks, artimetika interval,sifat distributif.

\section{PENDAHULUAN}

Suatu persamaan linear dalam $n$ variabel $x_{1}, x_{2}, \ldots, x_{n}$ adalah suatu persamaan dalam bentuk $a_{1} x_{1}+a_{2} x_{2}+\ldots+a_{n} x_{n}=b$ dengan $a_{i}=(i=1,2, \ldots, n)$ dan $b$ merupakan konstanta real. Sejumlah persamaan linear yang banyaknya berhingga dalam $n$ variabel $x_{1}, x_{2}, \ldots, x_{n}$ disebut Sistem Persamaan Linear (SPL) yang dapat ditulis dalam notasi persamaan matriks $A x=b$. Seiring dengan perkembangan ilmu matematika, koefisien - koefisien dan konstanta ruas kanan dari suatu SPL dapat berupa interval yang disebut dengan Sistem Persamaan Interval Linear (SPIL). Secara umum SPIL dapat ditulis dengan $\tilde{A} \tilde{x}=\tilde{b}$ dan dinyatakan juga sebagai perluasan dari SPL $A x=b$ dengan masing masing memenuhi $A \in \tilde{A}$ dan $b \in \tilde{b}$. Oleh karena itu, seperti pada SPL, untuk mencari solusi dari SPIL juga dapat menggunakan matriks yang disebut dengan matriks interval.

Matriks interval merupakan perluasan dari matriks real dengan elemen-elemen pada matriks interval berupa interval dan didefinisikan atas semua interval tertutup. Oleh karena itu, operasi aritmetika yang berlaku pada matriks interval juga menggunakan aturan-aturan yang berlaku dalam aritmetika interval.

Pada penelitian sebelumnya Hasen dan Smith [5] memulai penggunaan aritmetika interval dalam perhitungan matriks komputasi, beberapa penulis termotivasi dan terinspirasi seperti Alefeld dan Herzberger [6], Jaulin et al [7], Neumaier [8], Rohn [9] dan Ganesan dan Veeramani [10], telah mempelajari matriks interval.

Pada kehidupan nyata, ketidakakuratan pengukuran yang tak terhindarkan, yang tidak diketahui nilai pasti dari besaran yang diukur namun bisa mengetahui interval nilai yang mungkin dan oleh karena itu penggunaan matriks interval menjadi lebih tepat. Pada aritmetika interval ada sifat yang tidak selalu berlaku yaitu sifat distributif. Akibatnya, pada matriks interval, sifat - sifat yang terkait dengan sifat distributif tersebut juga tidak selalu berlaku. Untuk itu penulis pada penelitian ini tertarik untuk mengkaji operasi aritmetika pada matriks interval dan sifat - sifat operasi yang berlaku pada matriks interval. Pada penelitian ini, matriks interval yang dimaksud adalah matriks interval dengan elemen - elemennya berupa interval real.

\section{INTERVAL DAN BEBERAPA SIFATNYA}

Interval adalah himpunan bilangan-bilangan real yang ditunjukkan sebagai suatu pasangan berurut dan dinyatakan dalam suatu ketaksamaan [1]. Berikut akan diberikan definisi tentang interval tertutup. 
Definisi 1 [1] Interval tertutup adalah himpunan semua bilangan real $x$ yang dinyatakan dalam suatu ketaksamaan $\underline{x} \leq x \leq \bar{x}$ untuk sebarang konstanta real $\underline{x}$ dan $\bar{x}$ dengan $\underline{x} \leq \bar{x}$ dan dinotasikan dengan $[\underline{x}, \bar{x}]$ dan dapat dinyatakan kedalam notasi pembentuk himpunan sebagai berikut

$$
\tilde{x}=[\underline{x}, \bar{x}]=\{x \in \mathbb{R} \mid \underline{x} \leq x \leq \bar{x}\}
$$

dengan $\underline{x} \leq \bar{x}$ dan $\underline{x}, \bar{x} \in \mathbb{R}$.

Himpunan semua interval tertutup (IR) tersebut dapat dinyatakan dalam suatu notasi pembentuk himpunan sebagai berikut.

$$
\mathbf{I R}=\{\tilde{x} \mid \underline{x} \leq \bar{x}, \underline{x} \text { dan } \bar{x} \in \mathbb{R}\} .
$$

Dalam analisis interval, suatu ketaksamaan interval dinyatakan dalam bentuk interval pada garis real. Berikut ini digambarkan suatu interval pada garis bilangan real.

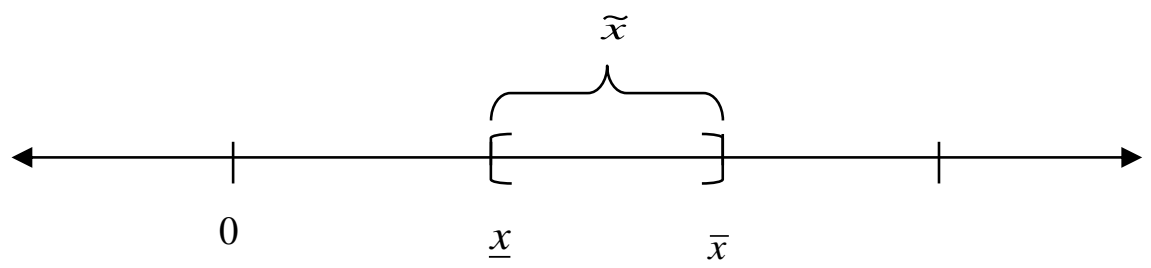

Gambar 1 Interval tertutup pada garis bilangan real beserta endpointsnya

Pada Gambar 1, titik $\underline{x}$ dan $\bar{x}$ disebut titik ujung (endpoints) interval. Titik $\underline{x}$ merupakan titik ujung (endpoints) yang terletak disebelah kiri dari suatu interval $\tilde{x}$ yang kemudian disebut sebagai lower endpoints (titik ujung bawah). Titik $\bar{x}$ merupakan titik ujung (endpoints) yang terletak disebelah kanan dari suatu interval $\tilde{x}$ yang kemudian disebut sebagai upper endpoints (titik ujung atas) sedemikian sehingga interval tersebut dapat ditulis $\tilde{x}=[\underline{x}, \bar{x}]$.

Jika * dinotasikan sebagai salah satu operasi untuk aritmetika pada bilangan real $x$ dan $y$, maka operasi yang sesuai untuk aritmetika pada interval $\tilde{x}$ dan $\tilde{y}$ adalah $\tilde{x} * \tilde{y}=\{x * y \mid x \in \tilde{x}, y \in \tilde{y}\}$. Untuk operasi $\tilde{x} * \tilde{y}$ dari dua interval $\tilde{x}=[\underline{x}, \bar{x}]$ dan $\tilde{y}=[\underline{y}, \bar{y}]$, maka sifat - sifat berikut berlaku:

\section{Definisi 2 [1]}

(1). Penjumlahan dari $\tilde{x}$ dan $\tilde{y}$ dapat dituliskan sebagai berikut:

$$
\tilde{x}+\tilde{y}=[\underline{x}+\underline{y}, \bar{x}+\bar{y}]
$$

(2). Pengurangan dari $\tilde{x}$ dan $\tilde{y}$ dapat dituliskan sebagai berikut:

$$
\tilde{x}-\tilde{y}=[\underline{x}-\bar{y}, \bar{x}-\underline{y}]
$$

(3). Perkalian dari $\tilde{x}$ dan $\tilde{y}$ dapat dituliskan sebagai berikut:

$$
\tilde{x} \cdot \tilde{y}=[\min (\underline{x} \underline{y}, \underline{x} \bar{y}, \bar{x} \underline{y}, \overline{x y}), \max (\underline{x} \underline{y}, \underline{x} \bar{y}, \bar{x} \underline{y}, \overline{x y})]
$$

(4). Pembagian dari $\tilde{x}$ dan $\tilde{y}$ dapat dituliskan sebagai berikut:

$$
\begin{gathered}
\tilde{x} \cdot \frac{1}{\tilde{y}}=\left[\min \left(\bar{x} \frac{1}{\bar{y}}, \bar{x} \frac{1}{y}, \underline{x} \frac{1}{\bar{y}}, \underline{x} \frac{1}{y}\right), \max \left(\bar{x} \frac{1}{\bar{y}}, \bar{x} \frac{1}{y}, \underline{x} \frac{1}{\bar{y}}, \underline{x} \frac{1}{y}\right)\right] \\
\text { dengan } \tilde{x} \div \tilde{y}=\tilde{x} \cdot \frac{1}{\tilde{y}} \text { dengan } 0 \in \tilde{y}
\end{gathered}
$$


Operasi pada aritmetika interval tidak memenuhi sifat distributif. Berikut akan diberikan contoh yang menunjukkan tidak berlakunya sifat distributif dalam aritmetika interval $(\tilde{x}(\tilde{y}+\tilde{z}) \neq \tilde{x} \tilde{y}+\tilde{x} \tilde{z})$.

Contoh 3 Diketahui: $\tilde{x}=[2,4], \tilde{y}=[2,2]$ dan $\tilde{z}=[-2,-2]$, maka:

$$
\begin{aligned}
\tilde{x}(\tilde{y}+\tilde{z}) & =[2,4]([2,2]+[-2,-2]) \\
& =[2,4] \cdot[0,0] \\
& =[0,0] \\
\tilde{x} \cdot \tilde{y}+\tilde{x} \cdot \tilde{z} & =[2,4] \cdot[2,2]+[2,4] \cdot[-2,-2] \\
& =[4,8]-[4,8] \\
& =[-4,4]
\end{aligned}
$$

Berdasarkan Contoh 3 , hasil operasi pada ruas kiri dan kanan tidak sama yaitu $\tilde{x}(\tilde{y}+\tilde{z}) \neq \tilde{x} \tilde{y}+\tilde{x} \tilde{z}$. Oleh karena itu, sifat distributif dipenuhi.

\section{MATRIKS INTERVAL DAN SIFAT-SIFATNYA}

Matriks interval merupakan himpunan dari matriks-matriks real dengan $a_{i j}$ yang menyatakan entri matriks pada baris ke- $i$ kolom ke-j dan untuk $\underline{a}_{i j}, \bar{a}_{i j} \in \mathbb{R}$ dengan $\underline{a}_{i j}$ adalah entri nilai lebih kecil dari $a_{i j}$ dan $\bar{a}_{i j}$ adalah entri nilai lebih besar dari $a_{i j}$. Secara umum, matriks interval dapat didefinisikan sebagai berikut:

Definisi 4 [2] Matriks interval Ã adalah suatu matriks dengan entri-entrinya berupa interval. Matriks interval Ã dapat dituliskan sebagai:

$$
\tilde{A}_{m \times n}=\left[\begin{array}{ccc}
\tilde{a}_{11} & \cdots & \tilde{a}_{1 n} \\
\vdots & \ddots & \vdots \\
\tilde{a}_{m 1} & \cdots & \tilde{a}_{m n}
\end{array}\right]=\left(\tilde{a}_{i j}\right)_{m \times n}
$$

dengan masing-masing $\tilde{a}_{i j}=\left[\underline{a}_{i j}, \bar{a}_{i j}\right], \underline{a}_{i j} \leq \bar{a}_{i j}$.

Selanjutnya, untuk menyatakan himpunan semua matriks interval berordo $m \times n$ digunakan notasi $(\boldsymbol{I R})^{m \times n}$. Hal ini berarti, untuk $\tilde{A} \in(\boldsymbol{I R})^{m \times n}, \tilde{A}=\left\{A=\left[a_{i j}\right] \mid \underline{a}_{i j} \leq a_{i j} \leq \bar{a}_{i j}, \underline{a}_{i j}\right.$ dan $\left.\bar{a}_{i j} \in \mathbb{R}\right\}$.

Pada Definisi 4, $\tilde{A}_{m \times n}$ adalah suatu matriks interval dengan $m$ menyatakan banyaknya baris dan $n$ menyatakan banyaknya kolom, $\tilde{a}_{i j}=\left[\underline{a}_{i j}, \bar{a}_{i j}\right]$ menyatakan entri matriks interval pada baris ke- $i$ kolom ke-j dengan $\underline{a}_{i j}$ menyatakan lower endpoint dari $\tilde{a}_{i j}$ dan $\bar{a}_{i j}$ menyatakan upper endpoint dari $\tilde{a}_{i j}$.

Pada aritmetika interval terdapat istilah midpoint dan width yang merupakan fungsi bernilai real dari suatu interval. Pada aritmetika matriks interval, midpoint dan width menyatakan fungsi bernilai real dari suatu matriks interval.

\section{Definisi 5 [3]}

a. Midpoint dari matriks interval $\tilde{A}$ disebut sebagai matriks midpoint dengan entri-entrinya didefinisikan sebagai:

$$
m(\tilde{A})=\left[\begin{array}{ccc}
m\left(\tilde{a}_{11}\right) & \cdots & m\left(\tilde{a}_{1 n}\right) \\
\vdots & \ddots & \vdots \\
m\left(\tilde{a}_{m 1}\right) & \cdots & m\left(\tilde{a}_{m n}\right)
\end{array}\right]
$$

b. Width dari matriks interval $\tilde{A}$ disebut sebagai matriks width dengan entri-entrinya didefinisikan sebagai 
c. $w(\tilde{A})=\left[\begin{array}{ccc}w\left(\tilde{a}_{11}\right) & \cdots & w\left(\tilde{a}_{1 n}\right) \\ \vdots & \ddots & \vdots \\ w\left(\tilde{a}_{m 1}\right) & \cdots & w\left(\tilde{a}_{m n}\right)\end{array}\right]$

dengan entri-entri matriks $w(\tilde{A})$ selalu nonnegatif.

Aritmetika matriks interval merupakan generalisasi dari aritmetika matriks real, dimana operasinya menggunakan aturan-aturan yang berlaku dalam aritmetika interval.

Jika " * " dinotasikan sebagai operasi aritmetika untuk menyatakan penjumlahan dan pengurangan pada matriks $A$ dan matriks $B$, maka operasi yang sesuai untuk aritmetika pada matriks interval $\tilde{A}$ dan matriks interval $\widetilde{B}$ didefinisikan sebagai berikut:

untuk $1 \leq i \leq m$ dan $1 \leq j \leq n$.

$$
\tilde{A} * \tilde{B}=\left\{\tilde{a}_{i j} * \tilde{b}_{i j} \mid \tilde{a}_{i j}, \tilde{b}_{i j} \in \mathbf{I R}\right\}
$$

Sedemikian sehingga operasi " * " yang memenuhi setiap operasi penjumlahan dan pengurangan pada matriks interval tersebut diperoleh definisi berikut:

Definisi 6 [2] Diberikan $\tilde{A}=\left[\tilde{a}_{i j}\right], \tilde{B}=\left[\tilde{b}_{i j}\right] \in(\mathbf{I R})^{\mathbf{m} \times \mathbf{n}}$ dengan $\tilde{a}_{i j}=\left[\underline{a}_{i j}, \bar{a}_{i j}\right]$, dan $\tilde{b}_{i j}=\left[\underline{b}_{i j}, \bar{b}_{i j}\right]$, untuk $1 \leq i \leq m$ dan $1 \leq j \leq n$ diperoleh:

1. Penjumlahan dari dua matriks interval $\tilde{A}$ dan $\tilde{B}$ dapat dituliskan sebagai berikut:

$$
[\tilde{A}+\tilde{B}]_{m \times n}=\left\{\left[\tilde{a}_{i j}+\tilde{b}_{i j}\right]_{m \times n} \mid \tilde{a}_{i j}, \tilde{b}_{i j} \in \mathbf{I R}\right\}
$$

2. Pengurangan antara dua matriks interval yaitu matriks interval Ã dan matriks interval $\tilde{B}$ adalah:

$$
[\tilde{A}-\tilde{B}]_{m \times n}=\left\{\left[\tilde{a}_{i j}-\tilde{b}_{i j}\right]_{m \times n} \mid \tilde{a}_{i j}, \tilde{b}_{i j} \in \mathbf{I R}\right\}
$$

Perkalian pada suatu matriks mensyaratkan banyaknya kolom pada matriks pertama harus sama dengan banyaknya baris pada matriks kedua. Oleh karena itu, operasi " * " yang didefinisikan untuk operasi penjumlahan dan operasi pengurangan tidak terpenuhi pada operasi perkalian matriks. Berikut diberikan definisi terkait perkalian pada matriks interval.

Definisi 7 [2] Diberikan $\tilde{A}=\left[\tilde{a}_{i j}\right] \in(\mathbf{I R})^{\mathbf{m} \times \mathbf{n}}$ dan $\tilde{C}=\left[\tilde{c}_{i j}\right] \in(\mathbf{I R})^{\mathbf{n} \times \mathbf{p}}, \tilde{\mathbf{x}} \in(\mathbf{I R})^{\mathbf{n}}$ dan $\tilde{\alpha} \in \mathbf{I R}$, diperoleh:

1. $\tilde{\alpha}[\tilde{A}]_{i j}=\left(\tilde{\alpha} \tilde{a}_{i j}\right)_{1 \leq i \leq m, 1 \leq j \leq n}$

2. $[\tilde{A} \tilde{C}]_{m \times p}=\left[\sum_{j=1}^{n}\left(\tilde{a}_{i j}\right)\left(\tilde{c}_{j k}\right)\right]_{1 \leq i \leq m, 1 \leq k \leq n}$

3. $\tilde{A} \tilde{\mathbf{x}}=\left(\sum_{j=1}^{n} \tilde{a}_{i j} \tilde{\mathbf{x}}\right)_{1 \leq i \leq m}$

Selanjutnya, berikut diberikan definisi tentang kekhususan matriks interval yang berhubungan dengan matriks midpoint dan matriks width.

\section{Definisi 8 [3]}

a. Dua matriks interval $\tilde{A}$ dan $\tilde{B}$ dikatakan ekuivalen dan dinotasikan dengan $\tilde{A} \approx \tilde{B}$ jika dan hanya jika matriks $m(\tilde{A})=m(\tilde{B})$.

b. Dua matriks interval $\tilde{A}$ dan $\tilde{B}$ dikatakan sama dan dinotasikan dengan $\tilde{A}=\tilde{B}$ jika dan hanya jika matriks $m(\tilde{A})=m(\tilde{B}) \operatorname{dan} w(\tilde{A})=w(\tilde{B})$. 
Pada matriks real untuk menyatakan matriks nol $\left[\begin{array}{ccc}0 & \cdots & 0 \\ \vdots & \ddots & \vdots \\ 0 & \cdots & 0\end{array}\right]$ digunakan notasi $O$ dan pada matriks interval untuk menyatakan matriks interval nol $\left[\begin{array}{ccc}\tilde{0} & \cdots & \tilde{0} \\ \vdots & \ddots & \vdots \\ \tilde{0} & \cdots & \tilde{0}\end{array}\right]$ digunakan notasi $\tilde{O}$. Berikut diberikan definisi yang berhubungan dengan matriks nol.

Definisi 9 [2] Misalkan $\tilde{A}$ adalah matriks interval $m \times n$, maka:

a. $\tilde{A}=\tilde{O}$ jika dan hanya jika $m(\tilde{A})=O$

b. $\tilde{A}=O$ jika dan hanya jika $m(\tilde{A})=O$ dan $w(\tilde{A})=O$.

c. $\tilde{A} \approx \tilde{O}$ jika dan hanya jika $m(\tilde{A})=O$ dan $w(\tilde{A}) \neq 0$.

d. $\tilde{A} \neq \tilde{O}$ jika dan hanya jika $\tilde{A}$ tidak ekuivalen dengan $\tilde{O}$.

Pada matriks real untuk menyatakan matriks identitas $\left[\begin{array}{ccc}1 & \cdots & 0 \\ \vdots & \ddots & \vdots \\ 0 & \cdots & 1\end{array}\right]$ digunakan notasi $I$ dan pada matriks interval untuk menyatakan matriks interval identitas $\left[\begin{array}{ccc}\tilde{1} & \cdots & \tilde{0} \\ \vdots & \ddots & \vdots \\ \tilde{0} & \cdots & \tilde{1}\end{array}\right]$ digunakan notasi $\tilde{I}$. Berikut diberikan definisi yang berhubungan dengan matriks identitas.

Definisi 10 [1] Misalkan Ã adalah matriks interval berordo $n \times n$, maka:

1. $\tilde{A}=\tilde{I}$ jika dan hanya jika $m(\tilde{A})=I$.

2. $\tilde{A}=I$ jika dan hanya jika $m(\tilde{A})=I$ dan $w(\tilde{A})=0$.

3. $\tilde{A} \approx \tilde{I}$ jika dan hanya jika $m(\tilde{A})=I \operatorname{dan} w(\tilde{A}) \neq 0$.

Pada matriks real ada beberapa sifat yang berlaku berdasarkan definisi operasi aritmetika yang terpenuhi pada matriks real. Akan tetapi, berdasarkan definisi operasi aritmetika pada matriks interval, sifat-sifat yang berlaku pada matriks real tidak semuanya berlaku pada matriks interval. Berikut diberikan teorema sifat-sifat yang berlaku pada matriks real yang juga berlaku pada matriks interval.

Teorema 11 [2] Untuk setiap matriks $\tilde{A}, \tilde{B}, \tilde{C} \in(\mathbf{I R})^{m \times n}$, aturan-aturan matriks berikut berlaku:
1. $\tilde{A}+\tilde{B}=\tilde{B}+\tilde{A}$
(Komutatif penjumlahan)

2. $\tilde{A}+(\tilde{B}+\tilde{C})=(\tilde{A}+\tilde{B})+\tilde{C} \quad$ (Asosiatif penjumlahan)

Bukti:

$$
\begin{aligned}
& \text { Misalkan } \tilde{A}=\left[\begin{array}{ccc}
\tilde{a}_{11} & \cdots & \tilde{a}_{1 n} \\
\vdots & \ddots & \vdots \\
\tilde{a}_{m 1} & \cdots & \tilde{a}_{m n}
\end{array}\right], \tilde{B}=\left[\begin{array}{ccc}
\tilde{b}_{11} & \cdots & \tilde{b}_{1 n} \\
\vdots & \ddots & \vdots \\
\tilde{b}_{m 1} & \cdots & \tilde{b}_{m n}
\end{array}\right], \tilde{C}=\left[\begin{array}{ccc}
c_{11} & \cdots & \tilde{c}_{1 n} \\
\vdots & \ddots & \vdots \\
\tilde{c}_{m 1} & \cdots & \tilde{c}_{m n}
\end{array}\right] \text {, maka: } \\
& \text { 1. } \tilde{A}+\tilde{B}=\left[\begin{array}{ccc}
\tilde{a}_{11} & \cdots & \tilde{a}_{1 n} \\
\vdots & \ddots & \vdots \\
\tilde{a}_{m 1} & \cdots & \tilde{a}_{m n}
\end{array}\right]+\left[\begin{array}{ccc}
\tilde{b}_{11} & \cdots & \tilde{b}_{1 n} \\
\vdots & \ddots & \vdots \\
\tilde{b}_{m 1} & \cdots & \tilde{b}_{m n}
\end{array}\right] \\
& =\left[\begin{array}{ccc}
\tilde{a}_{11}+\tilde{b}_{11} & \cdots & \tilde{a}_{1 n}+\tilde{b}_{1 n} \\
\vdots & \ddots & \vdots \\
\tilde{a}_{m 1}+\tilde{b}_{m 1} & \cdots & \tilde{a}_{m n}+\tilde{b}_{m n}
\end{array}\right]
\end{aligned}
$$




$$
\begin{aligned}
& =\left[\begin{array}{ccc}
\tilde{b}_{11}+\tilde{a}_{11} & \cdots & \tilde{b}_{1 n}+\tilde{a}_{1 n} \\
\vdots & \ddots & \vdots \\
\tilde{b}_{m 1}+\tilde{a}_{m 1} & \cdots & \tilde{b}_{m n}+\tilde{a}_{m n}
\end{array}\right] \\
& =\left[\begin{array}{ccc}
\tilde{b}_{11} & \cdots & \tilde{b}_{1 n} \\
\vdots & \ddots & \vdots \\
\tilde{b}_{m 1} & \cdots & \tilde{b}_{m n}
\end{array}\right]+\left[\begin{array}{ccc}
\tilde{a}_{11} & \cdots & \tilde{a}_{1 n} \\
\vdots & \ddots & \vdots \\
\tilde{a}_{m 1} & \cdots & \tilde{a}_{m n}
\end{array}\right] \\
& =\tilde{B}+\tilde{A}
\end{aligned}
$$

2. $\quad(\widetilde{B}+\widetilde{C})=\left[\begin{array}{ccc}\tilde{b}_{11} & \cdots & \tilde{b}_{1 n} \\ \vdots & \ddots & \vdots \\ \tilde{b}_{m 1} & \cdots & \tilde{b}_{m n}\end{array}\right]+\left[\begin{array}{ccc}c_{11} & \cdots & \tilde{c}_{1 n} \\ \vdots & \ddots & \vdots \\ \tilde{c}_{m 1} & \cdots & \tilde{c}_{m n}\end{array}\right]=\left[\begin{array}{ccc}\tilde{b}_{11}+\tilde{c}_{11} & \cdots & \tilde{b}_{1 n}+\tilde{c}_{1 n} \\ \vdots & \ddots & \vdots \\ \tilde{b}_{m 1}+\tilde{c}_{m 1} & \cdots & \tilde{b}_{m n}+\tilde{c}_{m n}\end{array}\right]$

$$
\begin{aligned}
\tilde{A}+(\tilde{B}+\tilde{C}) & =\left[\begin{array}{ccc}
\tilde{a}_{11} & \cdots & \tilde{a}_{1 n} \\
\vdots & \ddots & \vdots \\
\tilde{a}_{m 1} & \cdots & \tilde{a}_{m n}
\end{array}\right]+\left[\begin{array}{ccc}
\tilde{b}_{11}+\tilde{c}_{11} & \cdots & \tilde{b}_{1 n}+\tilde{c}_{1 n} \\
\vdots & \ddots & \vdots \\
\tilde{b}_{m 1}+\tilde{c}_{m 1} & \cdots & \tilde{b}_{m n}+\tilde{c}_{m n}
\end{array}\right] \\
& =\left[\begin{array}{ccc}
\tilde{a}_{11}+\left(\tilde{b}_{11}+\tilde{c}_{11}\right) & \cdots & \tilde{a}_{1 n}+\left(\tilde{b}_{1 n}+\tilde{c}_{1 n}\right) \\
\vdots & \ddots & \vdots \\
\tilde{a}_{m 1}+\left(\tilde{b}_{m 1}+\tilde{c}_{m 1}\right) & \cdots & \tilde{a}_{m n}+\left(\tilde{b}_{m n}+\tilde{c}_{m n}\right)
\end{array}\right] \\
& =\left[\begin{array}{ccc}
\left(\tilde{a}_{11}+\tilde{b}_{11}\right)+\tilde{c}_{11} & \cdots & \left(\tilde{a}_{1 n}+\tilde{b}_{1 n}\right)+\tilde{c}_{1 n} \\
\vdots & \ddots & \vdots \\
\left(\tilde{a}_{m 1}+\tilde{b}_{m 1}\right)+\tilde{c}_{m 1} & \cdots & \left(\tilde{a}_{m n}+\tilde{b}_{m n}\right)+\tilde{c}_{m n}
\end{array}\right] \\
& =\left[\begin{array}{cccc}
\tilde{a}_{11}+\tilde{b}_{11} & \cdots & \tilde{a}_{1 n}+\tilde{b}_{1 n} \\
\vdots & \ddots & \vdots \\
\tilde{a}_{m 1}+\tilde{b}_{m 1} & \cdots & \tilde{a}_{m n}+\tilde{b}_{m n}
\end{array}\right]+\left[\begin{array}{ccc}
\tilde{c}_{11} & \cdots & \tilde{c}_{1 n} \\
\vdots & \ddots & \vdots \\
\tilde{c}_{m 1} & \cdots & \tilde{c}_{m n}
\end{array}\right] \\
& =\left(\begin{array}{lll}
\tilde{A}+\tilde{B})+\tilde{C} \mathbf{\square}
\end{array}\right.
\end{aligned}
$$

Selanjutnya, diberikan contoh yang menunjukkan beberapa sifat yang berlaku pada matriks real tidak berlaku pada matriks interval yaitu sifat-sifat yang terkait dengan sifat distributif pada bilangan interval. Misalkan $\tilde{A} \in(\mathbf{I R})^{n \times p}, \tilde{B}, \tilde{C} \in(\mathbf{I R})^{m \times n}$ dan $\tilde{\alpha}, \tilde{\beta} \in \mathbf{I R}$ dengan

$$
\begin{aligned}
& \tilde{A}=\left[\begin{array}{cc}
{[-3,1]} & {[1,4]} \\
{[1,3]} & {[-2,2]} \\
{[2,5]} & {[-1,3]}
\end{array}\right], \quad \tilde{B}=\left[\begin{array}{ccc}
{[-1,2]} & {[2,4]} & {[-3,-1]} \\
{[3,5]} & {[-2,1]} & {[1,4]}
\end{array}\right], \quad \tilde{\alpha}=[-1,3], \quad \tilde{\beta}=[-1,3] \quad \text { dan } \\
& \tilde{C}\left[\begin{array}{ccc}
{[2,5]} & {[-1,3]} & {[3,5]} \\
{[-2,3]} & {[1,5]} & {[-3,2]}
\end{array}\right], \widetilde{D}=\left[\begin{array}{cc}
{[2,3]} & {[-3,1]} \\
{[-1,3]} & {[-2,2]}
\end{array}\right] \text {, }
\end{aligned}
$$

maka berdasarkan Definisi 6 dan 7 diperoleh:

$$
\begin{aligned}
& 2 \\
& ((\tilde{B}+\tilde{C}) \tilde{A})=\left[\begin{array}{ll}
{[-20,48]} & {[-17,54]} \\
{[-37,56]} & {[-23,56]}
\end{array}\right] \\
& ((\tilde{B}-\tilde{C}) \tilde{A})=\left[\begin{array}{ll}
{[-49,25]} & {[-58,18]} \\
{[-47,42]} & {[-21,63]}
\end{array}\right] \\
& \tilde{B} \tilde{A}+\tilde{C} \tilde{A}=\left[\begin{array}{ll}
{[-31,52]} & {[-30,60]} \\
{[-42,59]} & {[-32,64]}
\end{array}\right] \\
& \tilde{B} \tilde{A}-\tilde{C} \tilde{A}=\left[\begin{array}{ll}
{[-58,25]} & {[-62,28]} \\
{[-50,51]} & {[-33,63]}
\end{array}\right] \\
& 3 \\
& \tilde{A}(\tilde{B}+\tilde{C})=\left[\begin{array}{ccc}
{[-20,39]} & {[-25,31]} & {[-20,28]} \\
{[-15,37]} & {[-11,33]} & {[-12,24]} \\
{[-6,59]} & {[-4,53]} & {[-6,38]}
\end{array}\right] \quad \tilde{A} \tilde{B}+\tilde{A} \tilde{C}=\left[\begin{array}{ccc}
{[-26,40]} & {[-28,31]} & {[-29,38]} \\
{[-17,37]} & {[-15,35]} & {[-20,28]} \\
{[-12,59]} & {[-12,53]} & {[-21,41]}
\end{array}\right] \\
& 4 \\
& \tilde{A}(\tilde{B}-\tilde{C})=\left[\begin{array}{ccc}
{[-6,46]} & {[-43,5]} & {[-12,52]} \\
{[-32,14]} & {[-17,29]} & {[-38,10]} \\
{[-37,21]} & {[-26,32]} & {[-47,13]}
\end{array}\right] \quad \tilde{A} \tilde{B}-\tilde{A} \tilde{C}=\left[\begin{array}{lll}
{[-20,46]} & {[-43,16]} & {[-15,52]} \\
{[-34,20]} & {[-21,29]} & {[-38,10]} \\
{[-44,27]} & {[-32,33]} & {[-50,13]}
\end{array}\right] \\
& 5 \\
& \tilde{\alpha}(\tilde{B}+\tilde{C})=\left[\begin{array}{lll}
{[-7,21]} & {[-7,21]} & {[-4,12]} \\
{[-8,24]} & {[-6,18]} & {[-6,18]}
\end{array}\right] \quad \tilde{\alpha} \tilde{B}+\tilde{\alpha} \tilde{C}=\left[\begin{array}{lll}
{[-8,21]} & {[-7,21]} & {[-14,18]} \\
{[-11,24]} & {[-11,18]} & {[-13,18]}
\end{array}\right]
\end{aligned}
$$




$\begin{array}{ccccc}6 & \tilde{\alpha}(\tilde{B}-\tilde{C})=\left[\begin{array}{lll}{[-18,6]} & {[-5,15]} & {[-24,8]} \\ {[-7,21]} & {[-21,7]} & {[-7,21]}\end{array}\right] & \tilde{\alpha} \tilde{B}-\tilde{\alpha} \tilde{C}=\left[\begin{array}{ccc}{[-18,11]} & {[-13,15]} & {[-24,8]} \\ {[-14,21]} & {[-21,8]} & {[-10,21]}\end{array}\right] \\ 7 & (\tilde{\alpha}+\tilde{\beta}) \tilde{C}=\left[\begin{array}{ccc}{[0,35]} & {[-7,12]} & {[0,35]} \\ {[-14,21]} & {[0,35]} & {[-21,14]}\end{array}\right] & \tilde{\alpha} \tilde{C}+\tilde{\beta} \tilde{C}=\left[\begin{array}{ccc}{[-3,35]} & {[-7,21]} & {[-2,35]} \\ {[-14,21]} & {[-4,35]} & {[-21,14]}\end{array}\right] \\ 8 & (\tilde{\alpha}-\tilde{\beta}) \tilde{C}=\left[\begin{array}{lll}{[-25,10]} & {[-15,6]} & {[-25,10]} \\ {[-15,10]} & {[-25,10]} & {[-10,15]}\end{array}\right] & \tilde{\alpha} \tilde{C}-\tilde{\beta} \tilde{C}=\left[\begin{array}{ccc}{[-25,13]} & {[-15,13]} & {[-25,12]} \\ {[-18,17]} & {[-25,14]} & {[-17,18]}\end{array}\right] \\ 9 & \tilde{A}(\widetilde{D} \tilde{C})=\left[\begin{array}{cccc}{[-107,105]} & {[-84,130]} & {[-116,108]} \\ {[-57,105]} & {[-92,80]} & {[-42,114]} \\ {[-58,168]} & {[-129,127]} & {[-33,183]}\end{array}\right] & (\tilde{A} \widetilde{D}) \tilde{C}=\left[\begin{array}{lll}{[-99,126]} & {[-94,130]} & {[-116,109]} \\ {[-59,101]} & {[-80,80]} & {[-39,183]} \\ {[-61,162]} & {[-129,127]} & {[-46,114]}\end{array}\right]\end{array}$

Berdasarkan hasil yang diperoleh dari 1-9, terlihat bahwa beberapa sifat yang berlaku pada matriks real tidak berlaku pada matriks interval yaitu sifat-sifat yang terkait dengan sifat distributif pada bilangan interval. Akan tetapi berdasarkan penelitian yang telah dilakukan di [3] dan [4] sifat distributif interval yaitu $\tilde{x}(\tilde{y}+\tilde{z})=\tilde{x} \tilde{y}+\tilde{x} \tilde{z}$ akan berlaku:

a. Jika $\tilde{y} \tilde{z}>0$ atau $\tilde{y}$ dan $\tilde{z}$ adalah interval simetris.

b. Jika $\tilde{x}=[a, a]$ adalah interval degenerasi dengan $a>0$ dan $\tilde{y}, \tilde{z}$ adalah sebarang interval di IR.

c. Jika $\tilde{x}=[a, a]$ adalah interval degenerasi dengan $a=0$ dan $\tilde{y}, \tilde{z}$ adalah sebarang interval di IR.

d. Jika $\tilde{x}=[a, a]$ adalah interval degenerasi dengan $a<0$ dan $\tilde{y}, \tilde{z}$ adalah sebarang interval di IR.

Untuk kondisi-kondisi pada a-d yang dipenuhi oleh entri-entri matriks interval, mengakibatkan berlakunya beberapa sifat distributif untuk matriks interval.

Misal diberikan $\tilde{A}=\left[\begin{array}{ccc}{\left[\underline{a}_{11}, \bar{a}_{11}\right]} & \cdots & {\left[\underline{a}_{1 m}, \bar{a}_{1 m}\right]} \\ \vdots & \ddots & \vdots \\ {\left[\underline{a}_{p 1}, \bar{a}_{p 1}\right]} & \cdots & {\left[\underline{a}_{p m}, \bar{a}_{p m}\right]}\end{array}\right]$ dengan entri-entrinya $\tilde{A}$ adalah sebarang interval di IR, $\tilde{B}=\left[\begin{array}{ccc}{\left[-\bar{b}_{11}, \bar{b}_{11}\right]} & \cdots & {\left[-\bar{b}_{1 n}, \bar{b}_{1 n}\right]} \\ \vdots & \ddots & \vdots \\ {\left[-\bar{b}_{m 1}, \bar{b}_{m 1}\right]} & \cdots & {\left[-\bar{b}_{m n}, \bar{b}_{m n}\right]}\end{array}\right]$ dan $\tilde{C}=\left[\begin{array}{ccc}{\left[-\bar{c}_{11}, \bar{c}_{11}\right]} & \cdots & {\left[-\bar{c}_{1 n}, \bar{c}_{1 n}\right]} \\ \vdots & \ddots & \vdots \\ {\left[-\bar{c}_{m 1}, \bar{c}_{m 1}\right]} & \cdots & {\left[-\bar{c}_{m n}, \bar{c}_{m n}\right]}\end{array}\right]$ untuk setiap $\tilde{B}$ dan $\tilde{C}$ entri-entrinya adalah interval simetris dengan $\tilde{A} \in(\mathbf{I R})^{p \times m}, \operatorname{dan} \tilde{B}, \tilde{C} \in(\mathbf{I R})^{m \times n}$. Menggunakan Definisi 6 dan Definisi 7 :

1. Akan ditunjukkan bahwa $\tilde{A}(\tilde{B}+\tilde{C})=\tilde{A} \tilde{B}+\tilde{A} \tilde{C}$.

$$
\begin{aligned}
& \tilde{A}(\tilde{B}+\tilde{C})=\left[\begin{array}{ccc}
{\left[\underline{a}_{11}, \bar{a}_{11}\right]} & \cdots & {\left[\underline{a}_{1 m}, \bar{a}_{1 m}\right]} \\
\vdots & \ddots & \vdots \\
{\left[\underline{a}_{11}, \bar{a}_{p 1}\right]} & \cdots & {\left[\underline{a}_{m m}, \bar{a}_{p m}\right]}
\end{array}\right]\left(\left[\begin{array}{ccc}
{\left[-\bar{b}_{11}, \bar{b}_{11}\right]} & \cdots & {\left[-\bar{b}_{1 n}, \bar{b}_{1 n}\right]} \\
\vdots & \ddots & \vdots \\
{\left[-\bar{b}_{m 1}, \bar{b}_{m 1}\right]} & \cdots & {\left[-\bar{b}_{m n}, \bar{b}_{m n}\right]}
\end{array}\right]+\left[\begin{array}{ccc}
{\left[-\bar{c}_{11}, \bar{c}_{11}\right]} & \cdots & {\left[-\bar{c}_{1 n}, \bar{c}_{1 n}\right]} \\
\vdots & \ddots & \vdots \\
{\left[-\bar{c}_{m 1}, \bar{c}_{m 1}\right]} & \cdots & {\left[-\bar{c}_{m n}, \bar{c}_{m n}\right.}
\end{array}\right]\right) \\
& =\left[\begin{array}{c}
{\left[\left[-\left(\bar{a}_{11} \bar{b}_{11}\right)-\left(\bar{a}_{11} \bar{c}_{11}\right),\left(\bar{a}_{11} \bar{b}_{11}\right)+\left(\bar{a}_{11} \bar{c}_{11}\right)\right]+\cdots+\left[-\left(\bar{a}_{1 m} \bar{b}_{m 1}\right)-\left(\bar{a}_{1 m} \bar{c}_{m 1}\right),\left(\bar{a}_{1 m} \bar{b}_{m 1}\right)+\left(\bar{a}_{1 m} \bar{c}_{m 1}\right)\right]\right]} \\
\left.\vdots\left[-\left(\bar{a}_{p 1} \bar{b}_{11}\right)-\left(\bar{a}_{p 1} \bar{c}_{11}\right),\left(\bar{a}_{p 1} \bar{b}_{11}\right)+\left(\bar{a}_{p 1} \bar{c}_{11}\right)\right]+\cdots+\left[-\left(\bar{a}_{p m} \bar{b}_{m 1}\right)-\left(\bar{a}_{p m} \bar{c}_{m 1}\right),\left(\bar{a}_{p m} \bar{b}_{m 1}\right)+\left(\bar{a}_{p m} \bar{c}_{m 1}\right)\right]\right]
\end{array}\right. \\
& \cdots \quad\left[\left[-\left(\bar{a}_{11} \bar{b}_{1 n}\right)-\left(\bar{a}_{11} \bar{c}_{1 n}\right),\left(\bar{a}_{11} \bar{b}_{1 n}\right)+\left(\bar{a}_{11} \bar{c}_{1 n}\right)\right]+\cdots+\left[-\left(\bar{a}_{1 m} \bar{b}_{m n}\right)-\left(\bar{a}_{1 m} \bar{c}_{m n}\right),\left(\bar{a}_{1 m} \bar{b}_{m n}\right)+\left(\bar{a}_{1 m} \bar{c}_{m n}\right)\right]\right] \\
& \left.\left.\therefore \quad\left[-\left(\bar{a}_{p 1} \bar{b}_{1 n}\right)-\left(\bar{a}_{p 1} \bar{c}_{1 n}\right),\left(\bar{a}_{p 1} \bar{b}_{1 n}\right)+\left(\bar{a}_{p 1} \bar{c}_{1 n}\right)\right]+\cdots+\left[-\left(\bar{a}_{p m} \bar{b}_{m n}\right)-\left(\bar{a}_{p m} \bar{c}_{m n}\right),\left(\bar{a}_{p m} \bar{b}_{m n}\right)+\left(\bar{a}_{p m} \bar{c}_{m n}\right)\right]\right]\right]
\end{aligned}
$$

Kemudian pengoperasian di ruas kanan dilakukan dengan cara mengalikan terlebih dahulu kemudian baru dijumlahkan:

$$
\tilde{A} \tilde{B}=\left[\begin{array}{c}
{\left[\left[-\left(\bar{a}_{11} \bar{b}_{11}\right),\left(\bar{a}_{11} \bar{b}_{11}\right)\right]+\cdots+\left[-\left(\bar{a}_{1 m} \bar{b}_{m 1}\right),\left(\bar{a}_{1 m} \bar{b}_{m 1}\right)\right]\right]} \\
{\left[\left[-\left(\bar{a}_{p 1} \bar{b}_{11}\right),\left(\bar{a}_{p 1} \bar{b}_{11}\right)\right]+\cdots+\left[-\left(\bar{a}_{p m} \bar{b}_{m 1}\right),\left(\bar{a}_{p m} \bar{b}_{m 1}\right)\right]\right]}
\end{array}\right.
$$




$$
\begin{aligned}
& \cdots \quad\left[\left[-\left(\bar{a}_{11} \bar{b}_{1 n}\right),\left(\bar{a}_{11} \bar{b}_{1 n}\right)\right]+\cdots+\left[-\left(\bar{a}_{1 m} \bar{b}_{m n}\right),\left(\bar{a}_{1 m} \bar{b}_{m n}\right)\right]\right] \\
& \cdots \quad\left[\left[-\left(\bar{a}_{p 1} \bar{b}_{1 n}\right),\left(\bar{a}_{m 1} \bar{b}_{1 n}\right)\right]+\cdots+\left[-\left(\bar{a}_{p m} \bar{b}_{p m}\right),\left(\bar{a}_{p m} \bar{b}_{m n}\right)\right]\right] \\
& \tilde{A} \tilde{C}=\left[\begin{array}{c}
{\left[\left[-\left(\bar{a}_{11} \bar{c}_{11}\right),\left(\bar{a}_{11} \bar{c}_{11}\right)\right]+\cdots+\left[-\left(\bar{a}_{1 m} \bar{c}_{m 1}\right),\left(\bar{a}_{1 m} \bar{c}_{m 1}\right)\right]\right]} \\
\vdots \\
{\left[\left[-\left(\bar{a}_{p 1} \bar{c}_{11}\right),\left(\bar{a}_{p 1} \bar{c}_{11}\right)\right]+\cdots+\left[-\left(\bar{a}_{p m} \bar{c}_{m 1}\right),\left(\bar{a}_{p m} \bar{c}_{m 1}\right)\right]\right]}
\end{array}\right. \\
& \cdots \quad\left[\left[-\left(\bar{a}_{11} \bar{c}_{1 n}\right),\left(\bar{a}_{11} \bar{c}_{1 n}\right)\right]+\cdots+\left[-\left(\bar{a}_{1 m} \bar{c}_{m n}\right),\left(\bar{a}_{1 m} \bar{c}_{m n}\right)\right]\right] \\
& \because \quad \vdots \\
& \left.\cdots \quad\left[\left[-\left(\bar{a}_{p 1} \bar{c}_{1 n}\right),\left(\bar{a}_{p 1} \bar{c}_{1 n}\right)\right]+\cdots+\left[-\left(\bar{a}_{p m} \bar{c}_{m n}\right),\left(\bar{a}_{p m} \bar{c}_{m n}\right)\right]\right]\right] \\
& \tilde{A} \tilde{B}+\tilde{A} \tilde{C}=\left[\begin{array}{c}
{\left[\left[-\left(\bar{a}_{11} \bar{b}_{11}\right)-\left(\bar{a}_{11} \bar{c}_{11}\right),\left(\bar{a}_{11} \bar{b}_{11}\right)+\left(\bar{a}_{11} \bar{c}_{11}\right)\right]+\cdots+\left[-\left(\bar{a}_{1 m} \bar{b}_{m 1}\right)-\left(\bar{a}_{1 m} \bar{c}_{m 1}\right),\left(\bar{a}_{1 m} \bar{b}_{m 1}\right)+\left(\bar{a}_{1 m} \bar{c}_{m 1}\right)\right]\right.} \\
\vdots \\
{\left[-\left(\bar{a}_{p 1} \bar{b}_{11}\right)-\left(\bar{a}_{p 1} \bar{c}_{11}\right),\left(\bar{a}_{p 1} \bar{b}_{11}\right)+\left(\bar{a}_{p 1} \bar{c}_{11}\right)\right]+\cdots+\left[-\left(\bar{a}_{p m} \bar{b}_{m 1}\right)-\left(\bar{a}_{m n} \bar{c}_{m 1}\right),\left(\bar{a}_{m n} \bar{b}_{m 1}\right)+\left(\bar{a}_{m n} \bar{c}_{m 1}\right)\right]}
\end{array}\right] \\
& \cdots \quad\left[\left[-\left(\bar{a}_{11} \bar{b}_{1 n}\right)-\left(\bar{a}_{11} \bar{c}_{1 n}\right),\left(\bar{a}_{11} \bar{b}_{1 n}\right)+\left(\bar{a}_{11} \bar{c}_{1 n}\right)\right]+\cdots+\left[-\left(\bar{a}_{1 m} \bar{b}_{m n}\right)-\left(\bar{a}_{1 m} \bar{c}_{m n}\right),\left(\bar{a}_{1 m} \bar{b}_{m n}\right)+\left(\bar{a}_{1 m} \bar{c}_{m n}\right)\right]\right] \\
& \left.\cdots\left[\left[-\left(\bar{a}_{p 1} \bar{b}_{1 n}\right)-\left(\bar{a}_{p 1} \bar{c}_{1 n}\right),\left(\bar{a}_{p 1} \bar{b}_{1 n}\right)+\left(\bar{a}_{p 1} \bar{c}_{1 n}\right)\right]+\cdots+\left[-\left(\bar{a}_{p m} \bar{b}_{m n}\right)-\left(\bar{a}_{p m} \bar{c}_{m n}\right),\left(\bar{a}_{p m} \bar{b}_{m n}\right)+\left(\bar{a}_{p m} \bar{c}_{m n}\right)\right]\right]\right]
\end{aligned}
$$

Berdasarkan hasil yang diperoleh dari $\tilde{A}(\tilde{B}+\tilde{C})$ dan $\tilde{A} \tilde{B}+\tilde{A} \tilde{C}$, dapat disimpulkan bahwa $\tilde{A}(\tilde{B}+\tilde{C})=\tilde{A} \tilde{B}+\tilde{A} \tilde{C}$. Dengan cara yang sama, dapat diperoleh pula bahwa $\tilde{A}(\tilde{B}-\tilde{C})=\tilde{A} \tilde{B}-$ $\tilde{A} \tilde{C}$.

2. Akan ditunjukkan bahwa $\tilde{x}(\tilde{B}+\tilde{C})=\tilde{x} \tilde{B}+\tilde{x} \tilde{C}$.

$$
\begin{aligned}
& \tilde{x}(\tilde{B}+\tilde{C})=\left[\begin{array}{ccc}
{\left[\bar{x}\left(-\bar{b}_{11}-\bar{c}_{11}\right), \bar{x}\left(\bar{b}_{11}+\bar{c}_{11}\right)\right]} & \cdots & {\left[\bar{x}\left(-\bar{b}_{1 n}-\bar{c}_{1 n}\right), \bar{x}\left(\bar{b}_{1 n}+\bar{c}_{1 n}\right)\right]} \\
\vdots & \ddots & \vdots \\
{\left[\bar{x}\left(-\bar{b}_{m 1}-\bar{c}_{m 1}\right), \bar{x}\left(\bar{b}_{m 1}+\bar{c}_{m 1}\right)\right]} & \cdots & {\left[\bar{x}\left(-\bar{b}_{m n}-\bar{c}_{m n}\right), \bar{x}\left(\bar{b}_{m n}+\bar{c}_{m n}\right)\right]}
\end{array}\right] \\
& =\left[\begin{array}{ccc}
{\left[-\bar{x} \bar{b}_{11}-\overline{x c}_{11}, \bar{x} \bar{b}_{11}+\overline{x c}_{11}\right]} & \cdots & {\left[-\bar{x} \bar{b}_{1 n}-\overline{x c}_{1 n}, \bar{x} \bar{b}_{1 n}+\overline{x c}_{1 n}\right]} \\
\vdots & \ddots & \vdots \\
{\left[-\bar{x} \bar{b}_{m 1}-\overline{x c}_{m 1}, \bar{x} \bar{b}_{m 1}+\overline{x c}_{m 1}\right]} & \cdots & {\left[-\bar{x} \bar{b}_{m n}-\overline{x c}_{m n}, \bar{x} \bar{b}_{m n}+\overline{x c}_{m n}\right]}
\end{array}\right] \\
& \tilde{x} \tilde{B}+\tilde{x} \tilde{C}=\left[\begin{array}{ccc}
{\left[-\bar{x} \bar{b}_{11}, \bar{x} \bar{b}_{11}\right]} & \cdots & {\left[-\bar{x} \bar{b}_{1 n}, \bar{x} \bar{b}_{1 n}\right]} \\
\vdots & \ddots & \vdots \\
{\left[-\bar{x} \bar{b}_{m 1}, \bar{x} \bar{b}_{m 1}\right]} & \cdots & {\left[-\bar{x} \bar{b}_{m n}, \bar{x} \bar{b}_{m n}\right]}
\end{array}\right]+\left[\begin{array}{ccc}
{\left[-\overline{x c}_{11}, \overline{x c}_{11}\right]} & \cdots & {\left[-\overline{x c}_{1 n}, \overline{x x}_{1 n}\right]} \\
\vdots & \ddots & \vdots \\
{\left[-\overline{x c}_{m 1}, \overline{x c}_{m 1}\right]} & \cdots & {\left[-\overline{x c}_{m n}, \overline{x c}_{m n}\right]}
\end{array}\right] \\
& =\left[\begin{array}{ccc}
{\left[\left(-\bar{x} \bar{b}_{11}, \bar{x} \bar{b}_{11}\right)+\left(-\overline{x c}_{11}, \overline{x c}_{11}\right)\right]} & \cdots & {\left[\left(-\bar{x} \bar{b}_{1 n}, \bar{x} \bar{b}_{1 n}\right)+\left(-\overline{x c}_{1 n}, \overline{x c}_{1 n}\right)\right]} \\
\vdots & \ddots & \vdots \\
{\left[\left(-\bar{x} \bar{b}_{m 1}, \bar{x} \bar{b}_{m 1}\right)+\left(-\overline{x c}_{m 1}, \overline{x c}_{m 1}\right)\right]} & \cdots & {\left[\left(-\bar{x} \bar{b}_{m n}, \bar{x} \bar{b}_{m n}\right)+\left(-\overline{x c}_{m n}, \overline{x c}_{m n}\right)\right]}
\end{array}\right] \\
& =\left[\begin{array}{ccc}
{\left[-\bar{x} \bar{b}_{11}-\overline{x c}_{11}, \bar{x} \bar{b}_{11}+\overline{x c}_{11}\right]} & \cdots & {\left[-\bar{x} \bar{b}_{1 n}-\overline{x c}_{1 n}, \bar{x} \bar{b}_{1 n}+\overline{x c}_{1 n}\right]} \\
\vdots & \ddots & \vdots \\
{\left[-\bar{x} \bar{b}_{m 1}-\overline{x c}_{m 1}, \bar{x} \bar{b}_{m 1}+\overline{x c}_{m 1}\right]} & \cdots & {\left[-\bar{x} \bar{b}_{m n}-\overline{x c}_{m n}, \bar{x} \bar{b}_{m n}+\overline{x c}_{m n}\right]}
\end{array}\right]
\end{aligned}
$$

Jadi diperoleh bahwa $\tilde{x}(\tilde{B}+\tilde{C})=\tilde{x} \tilde{B}+\tilde{x} \tilde{C}$. Dengan cara yang sama, dapat diperoleh pula $\tilde{x}(\tilde{B}-\tilde{C})=\tilde{x} \tilde{B}-\tilde{x} \tilde{C}$.

3. Akan ditunjukkan bahwa $(\tilde{B}+\tilde{C}) \tilde{A}=\tilde{B} \tilde{A}+\tilde{C} \tilde{A}$. Pada kasus ini, diasumsikan $\tilde{A} \in(\mathbf{I R})^{n \times p}$, dan $\tilde{B}, \tilde{C} \in(\mathbf{I R})^{m \times n}$.

$$
(\tilde{B}+\tilde{C}) \tilde{A}=\left[\begin{array}{c}
{\left[\left(-\bar{b}_{11}-\bar{c}_{11}\right) \bar{a}_{11},\left(\bar{b}_{11}+\bar{c}_{11}\right) \bar{a}_{11}+\cdots+\left(-\bar{b}_{1 n}-\bar{c}_{1 n}\right) \bar{a}_{n 1},\left(\bar{b}_{1 n}+\bar{c}_{1 n}\right) \bar{a}_{n 1}\right]} \\
\vdots \\
{\left[\left(-\bar{b}_{m 1}-\bar{c}_{m 1}\right) \bar{a}_{11},\left(\bar{b}_{m 1}+\bar{c}_{m 1}\right) \bar{a}_{11}+\cdots+\left(-\bar{b}_{m n}-\bar{c}_{m n}\right) \bar{a}_{n 1},\left(\bar{b}_{m n}+\bar{c}_{m n}\right) \bar{a}_{n 1}\right]}
\end{array}\right.
$$




$$
\begin{aligned}
& \left.\begin{array}{cc}
\cdots & {\left[\left(-\bar{b}_{11}-\bar{c}_{11}\right) \bar{a}_{1 p},\left(\bar{b}_{11}+\bar{c}_{11}\right) \bar{a}_{1 p}+\cdots+\left(-\bar{b}_{1 n}-\bar{c}_{1 n}\right) \bar{a}_{m n},\left(\bar{b}_{1 n}+\bar{c}_{1 n}\right) \bar{a}_{m n}\right]} \\
\ddots & \vdots \\
\cdots & {\left[\left(-\bar{b}_{m 1}-\bar{c}_{m 1}\right) \bar{a}_{1 p},\left(\bar{b}_{m 1}+\bar{c}_{m 1}\right) \bar{a}_{1 p}+\cdots+\left(-\bar{b}_{m n}-\bar{c}_{m n}\right) \bar{a}_{n p},\left(\bar{b}_{m n}+\bar{c}_{m n}\right) \bar{a}_{n p}\right]}
\end{array}\right]
\end{aligned}
$$

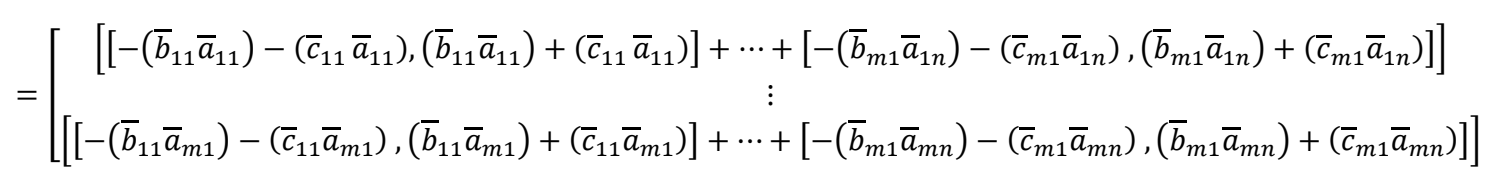

$$
\begin{aligned}
& \cdots \quad\left[\left[-\left(\bar{b}_{1 n} \bar{a}_{11}\right)-\left(\bar{c}_{1 n} \bar{a}_{11}\right),\left(\bar{b}_{1 n} \bar{a}_{11}\right)+\left(\bar{c}_{1 n} \bar{a}_{11}\right)\right]+\cdots+\left[-\left(\bar{b}_{m n} \bar{a}_{1 n}\right)-\left(\bar{c}_{m n} \bar{a}_{1 n}\right),\left(\bar{b}_{m n} \bar{a}_{1 n}\right)+\left(\bar{c}_{m n} \bar{a}_{1 n}\right)\right]\right] \\
& \left.\cdots \quad\left[-\left(\bar{b}_{1 n} \bar{a}_{m 1}\right)-\left(\bar{c}_{1 n} \bar{a}_{m 1}\right),\left(\bar{b}_{1 n} \bar{a}_{m 1}\right)+\left(\bar{c}_{1 n} \bar{a}_{m 1}\right)\right]+\cdots+\left[-\left(\bar{b}_{m n} \bar{a}_{m n}\right)-\left(\bar{c}_{m n} \bar{a}_{m n}\right),\left(\bar{b}_{m n} \bar{a}_{m n}\right)+\left(\bar{c}_{m n} \bar{a}_{m n}\right)\right]\right]
\end{aligned}
$$

$$
\begin{aligned}
& \tilde{B} \tilde{A}+\tilde{C} \tilde{A}=\left[\begin{array}{c}
{\left[\left[-\left(\bar{b}_{11} \bar{a}_{11}\right),\left(\bar{b}_{11} \bar{a}_{11}\right)\right]+\left[-\left(\bar{c}_{11} \bar{a}_{11}\right),\left(\bar{c}_{11} \bar{a}_{11}\right)\right]+\cdots+\left[-\left(\bar{b}_{m 1} \bar{a}_{1 n}\right),\left(\bar{b}_{m 1} \bar{a}_{1 n}\right)\right]+\left[-\left(\bar{c}_{m 1} \bar{a}_{1 n}\right),\left(\bar{c}_{m 1} \bar{a}_{1 n}\right)\right]\right]} \\
{\left[\left[-\left(\bar{b}_{11} \bar{a}_{m 1}\right),\left(\bar{b}_{11} \bar{a}_{m 1}\right)\right]+\left[-\left(\bar{c}_{11} \bar{a}_{m 1}\right),\left(\bar{c}_{11} \bar{a}_{m 1}\right)\right]+\cdots+\left[-\left(\bar{b}_{m 1} \bar{a}_{m n}\right),\left(\bar{b}_{m 1} \bar{a}_{m n}\right)\right]+\left[-\left(\bar{c}_{m 1} \bar{a}_{m n}\right),\left(\bar{c}_{m 1} \bar{a}_{m n}\right)\right]\right.}
\end{array}\right] \\
& \cdots \quad\left[\left[-\left(\bar{b}_{1 n} \bar{a}_{11}\right),\left(\bar{b}_{1 n} \bar{a}_{11}\right)\right]+\left[-\left(\bar{c}_{1 n} \bar{a}_{11}\right),\left(\bar{c}_{1 n} \bar{a}_{11}\right)\right]+\cdots+\left[-\left(\bar{b}_{m n} \bar{a}_{1 n}\right),\left(\bar{b}_{m n} \bar{a}_{1 n}\right)\right]+\left[-\left(\bar{c}_{m n} \bar{a}_{1 n}\right),\left(\bar{c}_{m n} \bar{a}_{1 n}\right)\right]\right] \\
& \cdots\left[\left[-\left(\bar{b}_{1 n} \bar{a}_{m 1}\right),\left(\bar{b}_{1 n} \bar{a}_{m 1}\right)\right]+\left[-\left(\bar{c}_{1 n} \bar{a}_{m 1}\right),\left(\bar{c}_{1 n} \bar{a}_{m 1}\right)\right]+\cdots+\left[-\left(\bar{b}_{m n} \bar{a}_{m n}\right),\left(\bar{b}_{m n} \bar{a}_{m n}\right)\right]+\left[-\left(\bar{c}_{m n} \bar{a}_{m n}\right),\left(\bar{c}_{m n} \bar{a}_{m n}\right)\right]\right] \\
& =\left[\begin{array}{c}
{\left[\left[-\left(\bar{b}_{11} \bar{a}_{11}\right)-\left(\bar{c}_{11} \bar{a}_{11}\right),\left(\bar{b}_{11} \bar{a}_{11}\right)+\left(\bar{c}_{11} \bar{a}_{11}\right)\right]+\cdots+\left[-\left(\bar{b}_{m 1} \bar{a}_{1 n}\right)-\left(\bar{c}_{m 1} \bar{a}_{1 n}\right),\left(\bar{b}_{m 1} \bar{a}_{1 n}\right)+\left(\bar{c}_{m 1} \bar{a}_{1 n}\right)\right]\right]} \\
{\left[\left[-\left(\bar{b}_{11} \bar{a}_{m 1}\right)-\left(\bar{c}_{11} \bar{a}_{m 1}\right),\left(\bar{b}_{11} \bar{a}_{m 1}\right)+\left(\bar{c}_{11} \bar{a}_{m 1}\right)\right]+\cdots+\left[-\left(\bar{b}_{m 1} \bar{a}_{m n}\right)-\left(\bar{c}_{m 1} \bar{a}_{m n}\right),\left(\bar{b}_{m 1} \bar{a}_{m n}\right)+\left(\bar{c}_{m 1} \bar{a}_{m n}\right)\right]\right.}
\end{array}\right] \\
& \cdots \quad\left[\left[-\left(\bar{b}_{1 n} \bar{a}_{11}\right)-\left(\bar{c}_{1 n} \bar{a}_{11}\right),\left(\bar{b}_{1 n} \bar{a}_{11}\right)+\left(\bar{c}_{1 n} \bar{a}_{11}\right)\right]+\cdots+\left[-\left(\bar{b}_{m n} \bar{a}_{1 n}\right)-\left(\bar{c}_{m n} \bar{a}_{1 n}\right),\left(\bar{b}_{m n} \bar{a}_{1 n}\right)+\left(\bar{c}_{m n} \bar{a}_{1 n}\right)\right]\right] \\
& \therefore\left[\left[-\left(\bar{b}_{1 n} \bar{a}_{m 1}\right)-\left(\bar{c}_{1 n} \bar{a}_{m 1}\right),\left(\bar{b}_{1 n} \bar{a}_{m 1}\right)+\left(\bar{c}_{1 n} \bar{a}_{m 1}\right)\right]+\cdots+\left[-\left(\bar{b}_{m n} \bar{a}_{m n}\right)-\left(\bar{c}_{m n} \bar{a}_{m n}\right),\left(\bar{b}_{m n} \bar{a}_{m n}\right)+\left(\bar{c}_{m n} \bar{a}_{m n}\right)\right]\right]
\end{aligned}
$$

Berdasarkan hasil yang diperoleh dari $(\tilde{B}+\tilde{C}) \tilde{A}$ dan $\tilde{B} \tilde{A}+\tilde{C} \tilde{A}$, dapat disimpulkan bahwa $\tilde{A}(\tilde{B}+\tilde{C})=\tilde{A} \tilde{B}+\tilde{A} \tilde{C}$. Dengan cara yang sama, dapat diperoleh pula bahwa $(\tilde{B}-\tilde{C}) \tilde{A}$ dan $\tilde{B} \tilde{A}-\tilde{C} \tilde{A}$.

Selain sifat-sifat pada 1-3, sifat lain yang terkait sifat distributif adalah sifat asosiatif perkalian pada matriks interval. Jika elemen-elemen pada matriks interval yang diberikan memenuhi kondisi-kondisi a-d, maka dengan cara yang sama seperti pada 1-3, dapat diperoleh pula bahwa sifat asosiatif perkalian matriks interval dipenuhi.

\section{KESIMPULAN}

Berdasarkan penelitian yang telah dilakukan dapat disimpulkan bahwa beberapa sifat dari matriks real berlaku pada matriks interval yaitu sifat komutatif dan asosiatif pada penjumlahan. Akan tetapi terdapat pula beberapa sifat yang tidak berlaku khususnya yang terkait dengan sifat distributif $\tilde{x}(\tilde{y}+\tilde{z})=\tilde{x} \tilde{y}+\tilde{x} \tilde{z}$ pada bilangan interval. Sifat-sifat tersebut secara khusus akan berlaku apabila entri-entri matriks interval memenuhi:

a. Jika $\tilde{y} \tilde{z}>0$ atau $\tilde{y}$ dan $\tilde{z}$ adalah interval simetris.

b. Jika $\tilde{x}=[a, a]$ adalah interval degenerasi dengan $a>0$ dan $\tilde{y}, \tilde{z}$ adalah sebarang interval di IR.

c. Jika $\tilde{x}=[a, a]$ adalah interval degenerasi dengan $a=0$ dan $\tilde{y}, \tilde{z}$ adalah sebarang interval di IR.

d. Jika $\tilde{x}=[a, a]$ adalah interval degenerasi dengan $a<0$ dan $\tilde{y}, \tilde{z}$ adalah sebarang interval di IR 


\title{
DAFTAR PUSTAKA
}

[1]. Hansen E. Interval Arithmetic With Some Applications For Digital Computers. 1965.

[2]. Ganesan, K. On Some Properties Of Interval Matrices. J Comp Mathematical Science. 2007; $1: 2$.

[3]. Nirmala T, Datta D, Kushwaha H S. Invers Interval Matrix: A New Approach. J Mathematical Sciences. 2011; 5: 607-624.

[4]. Chiou P K. Inclusion Monotonic Property Of Courant-Fischer Minimax Characterization On Interval Eigen Problems For Symmetric Interval Matrices. Journal Of Mathematical Sciences. 1999; 11-22.

[5]. E. R. Hansen and R. R. Smith, "Interval arithmetic in matrix computations", Part 2, SI AM. Journal of Numerical Analysis, vol. 4, pp. 1-9, 1967.

[6]. G. Alefeld and J. Herzberger, 'Introduction to Interval Computations", Academic Press, New York, 1983.

[7]. Luc Jaulin, Michel Kieffer, Olivier Didrit and Eic Walter, "Applied Inteval Analysis", Springer-Verlag, London, 2001.

[8]. A. Neumaier, "Interval Methods for Systems of Equations", Cambridge University Press, Cambridge, 1990.

[9]. J. Rohn, "Interval matrices: singularity and real eigenvalues", SIAM. Journal of Matrix Analysis and Applications, vol. 1, pp. 82 - 91, 1993.

[10]. K. Ganesan and P. Veeramani, "On Arithmetic Operations of Interval Numbers", International Journal of Unccertainty, Fuzziness and Knowledge - Based Systems, vol. 13, no. 6, pp. $619-631,2005$.

\author{
EPIFANIA KURVA $\quad$ : Jurusan Matematika FMIPA UNTAN, Jl. Jend. A. Yani \\ Pontianak, epifaniakurva9@gmail.com \\ EVI NOVIANI $\quad$ : Jurusan Matematika FMIPA UNTAN, Jl. Jend. A. Yani \\ Pontianak, evi_noviani@math.untan.ac.id \\ NILAMSARI KUSUMASTUTI : Jurusan Matematika FMIPA UNTAN, Jl. Jend. A. Yani \\ Pontianak, nilamkusumastuti@math.untan.ac.id
}

